HStud 25 (2011)1, 25-36

DOI: 10.1556/HStud.25.2011.1.2

\title{
TRADUCTION DES TEXTES À CONTRAINTE : MÉTHODES ET EXPÉRIENCES HONGROISES
}

\author{
LEVENTE SELÁF \\ Université Eötvös Loránd \\ Budapest, Hongrie
}

\begin{abstract}
L'Oulipo est un groupe littéraire né en 1960 dont le principe est l'écriture de textes littéraires à contraintes. L'auteur de cet article présente les conceptions oulipiennes de la littérature, qui ne sont pas sans relations avec la problématique de la traduction. De la traduction poétique, en particulier, puisque celle-ci doit faire face à toutes sortes de contraintes imposées par la forme de l'original à traduire. On présente aussi brièvement la comparaison de la pratique de la traduction poétique en France et en Hongrie. En finissant avec le cas concret de traductions de certains textes oulipiens, notamment ceux de Jacques Roubaud en hongrois.
\end{abstract}

Mots-clefs : Oulipo, textes à contraintes, traduction poétique, Jacques Roubaud

L'Oulipo, fondé en 1960, appartient probablement au nombre des cercles littéraires les plus considérables. ${ }^{1}$ Les oulipiens, Raymond Queneau, Georges Perec, Italo Calvino, Marcel Duchamp, Oskar Pastior, Jacques Roubaud, pour ne citer que quelques-uns des plus connus, ont adopté pour principe l'écriture d'ouvrages littéraire observant des contraintes, des règles établies par eux-mêmes et dont ils ont démontré le potentiel créateur ; l'Oulipo a ainsi enrichi de plusieurs chefsd'œuvre la littérature mondiale au cours d'un demi-siècle.

Dans cet article, nous souhaitons faire le bilan momentané de la traduction hongroise des textes oulipiens et donner un aperçu des différentes méthodes possibles employées lors de ces traductions. Pour cela, nous souhaitons d'abord esquisser très brièvement l'histoire de la traduction hongroise et française dans ses rapports avec la forme des œuvres traduites, puis présenter les conceptions de la traduction de la poétique de l'Oulipo en finissant par quelques exemples de traductions potentielles (hongroises ou non) pour des textes oulipiens.

Les réflexions sur la traduction littéraire se rapportent souvent, d'une manière ou d'une autre, à l'Oulipo : parfois les oulipiens sont mentionnés comme créateurs de textes intraduisibles ou pour le moins très difficiles à traduire, ce qui peut servir de point de départ aux discussions sur la traductibilité des textes littéraires en gé- 
néral ; il arrive aussi que les textes composés à partir des contraintes fixées par l'Oulipo présentent une analogie avec des traductions, c'est-à-dire que les transformations subies par un texte au cours du travail oulipien sont comparables à la transposition d'une langue à une autre. ${ }^{2}$ D'ailleurs, les oulipiens ont également créé des contraintes qui sont elles-mêmes définies comme «traductions ».

Nous savons que l'Oulipo a divisé l'histoire littéraire en deux périodes : celle qui précède et celle qui suit la fondation de l'ouvroir en 1960. Notons que les contraintes et les règles formelles inventées avant 1960 (les anoulipismes) sont librement réutilisées, remodelées par les membres du groupe. L'Oulipo est à la fois une chambre de rhétorique illustrant à merveille le pouvoir créateur d'un art tombé en désuétude et absent des programmes de l'Éducation nationale et un cercle littéraire cherchant avec assiduité des nouvelles formes d'expression pour la littérature. Il est certain que le jeu systématique des oulipiens avec les formes et avec la langue impose à ceux qui lisent et traduisent leurs textes de s'engager dans une réflexion sur le caractère essentiel ou accidentel des contraintes. Que faut-il transmettre dans une traduction et quel est le rôle joué par les contraintes formelles? La traduction des textes oulipiens montre une analogie très claire avec la traduction des poèmes, textes à contraintes formelles souvent précises et fréquemment à forme (plus ou moins) fixe ; il suffit de penser aux distiques, hexamètres, sonnets, ballades, rondeaux, aux chansons des troubadours ou aux haïkaï japonais, et ainsi de suite.

Bien sûr, certaines contraintes déterminent la structure narrative et n'affectent pas particulièrement la traduction : les règles quotidiennes de l'usage de la langue ne sont point changées dans les textes au sein desquels on les applique. Ainsi « la contrainte de Pascal » ou encore « X prend Y pour $Z »{ }^{3}$ Mais plus fréquentes sont celles dont l'application sollicite, tout comme les poèmes d'une forme particulièrement difficile, une traduction qui respecte la forme. La complexité de la structure formelle est une sorte de défi lancé aux traducteurs, au moins à certaines époques et dans le cas de certaines littératures, y compris dans la vie littéraire hongroise contemporaine.

Si nous voulons présenter les difficultés attachées à la traduction des textes oulipiens en hongrois, nous devons d'abord examiner les méthodes hongroises de la traduction des textes à contrainte formelle autres que les textes oulipiens.

\section{Pratique de la traduction poétique en Hongrie}

Dans les premiers temps de la littérature hongroise, les traducteurs ne cherchaient pas particulièrement à reproduire la forme de l'original. La poésie était dominée par une versification strophique très simple, employant des strophes isorimiques, isométriques qui donnaient un cadre à la réécriture des compositions 
très diverses, des nouvelles de Boccace jusqu'à des poèmes pétrarquistes en latin. Bien que les lettrés du $16^{\mathrm{e}}$ siècle n'aient pas tardé à remarquer qu'il était techniquement possible de construire des mètres gréco-latins dans un texte hongrois, les mètres antiques furent expérimentés d'abord au sein d'œuvres personnelles rédigées directement en hongrois à l'imitation des poèmes antiques. ${ }^{4}$ Ce n'est que progressivement que la reconstruction exacte des mètres devint essentielle pour les traducteurs qui se voulaient fidèle à l'original. On mentionne Benedek Virág (1752-1830) comme le premier qui eût traduit des poèmes antiques en tenant compte non seulement du fond, mais aussi de la forme. ${ }^{5}$ Parmi les premiers essais de reproduction de la forme originale, on peut citer la version hongroise des psaumes de Théodore de Bèze et de Clément Marot proposée par Albert Szenci Molnár (1574-1634), réalisée à partir d'une traduction allemande accomplie par Ambrosius Lobwasser. Dans sa préface, Szenci exprima clairement la raison de ses efforts pour maintenir la structure syllabique et la succession des rimes telle qu'elles se présentaient dans la version allemande, tout en ajoutant qu'en fin de compte, pour lui, le contenu proprement dit prévalait sur l'intention formelle. ${ }^{6}$ Les premiers débats importants sur la façon de traduire éclatèrent en Hongrie dans la première moitié du $19^{\mathrm{e}}$ siècle. À cette époque, la pratique et le discours théorique de la traduction étaient notamment marqué par la tradition française du $17^{\mathrm{e}}$ siècle. Nombreux étaient les partisans des « belles infidèles » qui, considérant la traduction poétique comme une création, ne revendiquaient pas la conservation de la forme ; ils souhaitaient rendre le contenu littéraire dans une langue hongroise claire, fluide, compréhensible. Peu à peu, toujours au $19^{\mathrm{e}}$ siècle, nous observons toutefois l'acclimatation en Hongrie d'une tendance apparue en Allemagne, celle d'une forte exigence de fidélité à la forme. C'est ainsi qu'à la fin du siècle, déjà, on pouvait esquisser une histoire téléologique de la traduction hongroise décrivant une évolution vers l'observation rigoureuse des contraintes formelles. ${ }^{7}$

En Hongrie, la traduction des sonnets, des strophes grecques et latines, des Lieder allemands était conçue comme une forme de naturalisation de structures raffinées, absentes de la tradition littéraire hongroise. C'était un programme de renouveau conscient. Il fallait créer des équivalents qui pour la vue et pour l'ouïe ressemblassent suffisamment aux originaux. L'histoire de la traduction des Fleurs $d u$ Mal de Baudelaire est l'exemple le plus célèbre de la conquête parallèle d'une forme et d'une esthétique nouvelles, qui s'achève avec la traduction à trois voix de Mihály Babits, Árpád Tóth et Lőrinc Szabó, publiée en 1923, restée un modèle de "fidélité" à la forme.

En réalité, la reproduction de la forme originale signifie, selon ce système cristallisé au cours d'une pratique centenaire, l'adoption d'un système d'équivalence métrique : on a créé l'équivalent de presque tous les vers et de toutes les strophes naturalisés. ${ }^{8}$ L'alexandrin, par exemple, est reproduit par un dodécasyllabe avec césure au milieu, terminé par un iambe, ou bien, s'il s'agit d'une rime féminine, 
par un trochée qui ajoute une septième syllabe surnuméraire au deuxième hémistiche. Cette manière s'est imposée à tous dans la deuxième moitié du $20^{\mathrm{e}}$ siècle, où la ressemblance formelle a commencé à dépasser en importance l'identité de la signification. ${ }^{9}$ Il ne serait pas étonnant qu'une solution particulièrement bien réussie du point de vue du contenu soit écartée quand elle n'entre pas dans les cadres de la forme imitative très strictement définie. Ce style classique, très formel, presque ritualisé s'est imposé pour la traduction des formes traditionnelles de la poésie européenne, tandis que la poésie, de son côté, évoluait librement, en parallèle avec la pratique poétique mondiale. Ce conservatisme de la traduction a permis la création d'œuvres d'art d'une valeur exceptionnelle, des copies ou maquettes d'une extraordinaire ressemblance avec les originaux. D'ailleurs, le travail du traducteur de poésie a acquis (et conservé jusqu'à une époque très récente) un grand prestige social ; envisagée de cette manière, l'activité du traducteur ressemble beaucoup à la création selon des règles formelles sévères, telle que la pratiquent les auteurs oulipiens.

À l'heure actuelle, le souci de la fidélité formelle tend à disparaitre ; l'exemple le plus éloquent en est la nouvelle traduction de la Divine Comédie par Ádám Nádasdy. Ce dernier, s'abstenant d'utiliser la rime et choisissant de s'exprimer en vers blancs - forme qui correspond à sa formation d'angliciste et à son goût littéraire -, affirme que cette méthode est plus contemporaine qu'une version rimée, à une époque où la poésie renonce de plus en plus à ce procédé rhétorique.

Il est pour le moins étrange que l'Oulipo ne bénéficie pas de cette estime dans laquelle on continue tout de même à tenir, en Hongrie, la poésie à contraintes fortes (forme et contenu déterminés par l'original). Du moins, cela ne semble pas se refléter dans la réception de ses ouvrages. Avant de présenter la pratique actuelle des traducteurs hongrois concernant les textes oulipiens, il apparaît opportun d'esquisser les méthodes de traduction que nous suggère les activités de l'Oulipo.

\section{Les méthodes de traduction oulipiennes}

Plusieurs membres de l'Oulipo ont embrassé la traduction littéraire (parfois celle des textes de leurs confrères oulipiens), ce qui montre peut-être la voie qu'il faut suivre lorsqu'il s'agit de traduire leurs œuvres. Jacques Roubaud, par exemple, en traduisant les cansos des troubadours occitans, a renoncé à l'usage de la rime et, dans la plupart des cas, n'a pas non plus conservé les nombres de syllabes de l'original. D'autre part, pour refléter l'autonomie des coblas (strophes) de la forme originale, il n'a pas adopté la pratique moderne qui consiste à commencer un nouvel alinéa à la fin de chaque vers. Il a suivi l'exemple des chansonniers médiévaux qui inséraient un point entre les vers d'une même strophe ; mais en remplaçant ces points par des blancs longs, tels qu'il les utilise souvent dans sa propre 
poésie. De même, en traduisant des tankas japonaises ou des livres de la Bible, il a essayé de rendre l'effet visuel produit par le texte écrit original.

Harry Mathews a proposé toute une série de techniques de la traduction selon la conception des oulipiens : traductions antonymique, grammaticale, homoléxique, homophonique, homosémantique, sémodéfinitionnelle, translexicale et ainsi de suite. ${ }^{10}$ Chacune de ces méthodes envisage d'une manière particulière la restitution du contenu ou de la forme d'un texte original, bien sûr jamais comme le ferait une traduction littéraire usuelle. Un texte source est une potentialité à explorer pour créer un (ou plusieurs) autre(s) texte(s). Le nombre de lettres contenues dans l'original, la définition de ses mots, la syntaxe du texte, etc. offrent des voies très variées au traducteur : il n'existe pas de hiérarchie parmi les contraintes ou au sein des aspects propres à un texte à traduire.

L'approche de Mathews reflète la méfiance absolue envers la traduction quant à la transmission du sens : comment croire à sa réussite quand on constate, dans une seule et même langue, que la paraphrase altère le sens et que les solutions potentielles peuvent difficilement être classées dans un ordre hiérarchique.

\section{Traduire des textes oulipiens}

Parmi les contraintes inventées ou renouvelées par l'Oulipo, certaines ont plus d'influence sur la traduction que d'autres. Celles qui concernent la composition narrative sont neutres, du point de vue de la traduction, et n'exigent pas des traducteurs un travail supplémentaire. ${ }^{11}$ D'ailleurs, les bibines, terines, quinines et autres formes fixes ne sont pas plus compliquées à traduire que les autres formes de poésie, plus traditionnelles, auxquelles est généralement confronté le traducteur de poésie. Il existe, en revanche, des contraintes visibles, apparentes qu'un traducteur rencontre plus rarement comme les avalanches ou les avions (inventés par les oulipiens), les palindromes ou les anagrammes (reliques du passé littéraire). Un exemple fréquemment cité à cause de sa difficulté formelle est La Disparition de Georges Perec, conçue selon les règles du lipogramme. La traduction des textes lipogrammatiques de Georges Perec est particulièrement difficile : il s'agit d'une contrainte spectaculaire et incontournable qui demande un grand effort de la part du traducteur. D'ailleurs, les solutions ne sont pas identiques à chaque tentative, compte tenu, en particulier, des spécificités propres à chaque langue destinataire. Par exemple, la traduction japonaise doit substituer à la contrainte originale (l'absence de la lettre e) un équivalent proche, du fait que le japonais n'utilise pas l'alphabet latin mais une écriture syllabique. ${ }^{12}$ Mais les solutions diffèrent aussi selon les conceptions adoptées par les traducteurs. En étudiant deux traductions en anglais de La Disparition, Alison James a caractérisé les deux méthodes employées qui se distinguent, d'une part, par leurs références culturel- 
les (dans le premier cas, ces dernières sont identiques à celles du texte source et renvoient donc à des realia inconnus du lecteur anglophone, dans le second cas, elles sont remplacées par des équivalents qui sont tout à fait familiers aux anglo-saxons) ; d'autre part, par l'absence ou la présence de l'autoréflexion dans la traduction (à propos des périphrases rendues nécessaires pour obéir à la contrainte). L'un des traducteurs, Gilbert Adair, a modifié l'original assez librement afin de respecter la contrainte de base ; proposant une « traduction mot-à-mot », il a rendu le texte dans un ton familier à ses lecteurs. Le second traducteur, Lee, au contraire, est allé jusqu'à se permettre de commenter l'original et son propre travail de traducteur (toujours en évitant l'usage des e) quand cela lui a paru nécessaire. ${ }^{13}$ Ces amplifications de Lee rapprochent sa méthode de la « traduction littérale » selon la définition de Berman, toujours d'après James. ${ }^{14}$

Une application créative de la contrainte qui permet d'obtenir un ouvrage de qualité dans la langue de destination est absolument conforme à la conception de la littérature qui est celle de l'Oulipo. Le traducteur des textes oulipiens est en droit d'exploiter les possibilités offertes par la contrainte adoptée par l'auteur. Si la langue source l'avait permis, ce dernier y aurait selon toute probabilité recouru.

La traduction des textes oulipiens concerne avant tout des textes composés en français, ce qui pourrait théoriquement avoir une influence sur la méthode mise en œuvre lors de leur traduction, c'est-à-dire que les traducteurs pourraient se conformer à une certaine pratique française de la traduction littéraire. Or, la reproduction fidèle de la forme ne s'inscrit pas dans cette tradition dite française. Il est au contraire assez rare que le traducteur français se donne pour objectif de respecter la forme ; certes, nous en avons quelques exemples, relativement anciens, comme la traduction du Chansonnier de Pétrarque par Vasquin Philieul (1548), qui a traduit les sonnets en sonnets ; toutefois il a remplacé l'endecasillabo par des décasyllabes, et il a parfois modifié la structure des rimes. Sa fidélité se fait voir dans la reproduction soigneuse de la structure des sextines de Pétrarque, composant ainsi les premiers spécimens de cette forme poétique en langue française. Plus tard, au $17^{\mathrm{e}}$ siècle, apparurent en France les traductions qui, même pour ce qui est du fond, argumentent en faveur d'une réécriture embellissant l'original. À l'époque classique, la sévérité des règles de la prosodie en matière de traduction poétique n'aurait pas permis la traduction de bien des poèmes dans leur forme originale ; l'usage constant de l'alexandrin et l'alternance des rimes masculines et féminines étaient recommandées aux traducteurs autant qu'aux auteurs. C'est à la fin du $19^{\mathrm{e}}$ siècle, au moment où les principes de la traduction littéraire étaient instaurés en Hongrie, que se produisit la « crise du vers » dans la poésie française. La tyrannie des formes traditionnelles, sacralisées, fut abolie par le vers libre, le poème en prose, la mise en page novatrice. Néanmoins, à cette époque, en France, le rapport à la traduction poétique n'en fut pas pour autant changé, on n'exigea pas plus qu'avant l'observation rigoureuse de la forme poétique de l'original. 
Les traducteurs hongrois des textes oulipiens n'ont pas adopté cette pratique française, de même qu'ils ont renoncé aux solutions offertes par la théorie de la traduction de l'Oulipo.

\section{L'Oulipo en Hongrie}

Comme le dit Hervé Le Tellier, « toute traduction d'une œuvre oulipienne est un exploit et une œuvre oulipienne en soi $» .{ }^{15}$ Or il nous paraît que peu nombreux sont les traducteurs hongrois ayant voulu créer des œuvres oulipiennes à partir des œuvres d'oulipiens. L'art caractérisé par des normes applicables à la forme, élaboré pour la traduction restait essentiellement réservé à la poésie.

En réalité, très peu d'ouvrages oulipiens à contrainte sont traduits en hongrois. En ce qui concerne la traduction des textes lipogrammatiques et anagrammatiques de Perec, les traducteurs hongrois ont opté pour une solution très commode, ${ }^{16}$ c'est-à-dire de les ignorer complètement. ${ }^{17} \mathrm{D}$ 'ailleurs, la littérature hongroise ne méconnaît pas ces procédés, mais leur usage reste anecdotique et restreint à la sphère des poèmes de circonstance. ${ }^{18}$

Nous pouvons compter, parmi les ouvrages de grande haleine traduits à ce jour, Les Exercises de style de Raymond Queneau, Le Grand Incendie de Londres et Quelque chose noir de Jacques Roubaud, plusieurs romans d'Italo Calvino et de Georges Perec, un recueil de récits de Paul Fournel. Dans les revues littéraires ou les anthologies, on trouve encore, éparpillés, des poèmes ou proses brèves de Jacques Jouet, de Marcel Duchamp et d'Oskar Pastior, sans que cela reflète pour autant un programme de traduction réfléchi. András Petőcz a commencé une traduction des Cent mille milliards de poèmes de Raymond Queneau, mais, à notre connaissance, l'entreprise n'a jamais été achevée, faute de temps probablement. En ce qui concerne Calvino, on ne mentionne quasiment jamais qu'il a composé une grande partie de son œuvre à partir de contraintes et en tant que membre de l'Oulipo : il est aussi vrai que ses recherches poétiques concernaient plus les procédés narratifs que l'expression proprement dite, ainsi leur traduction ne reflète-t-elles pas nécessairement les contraintes formelles propres aux ouvrages difficilement traduisibles de l'Oulipo.

Dans sa traduction des Exercices de style, Róbert Bognár a observé une méthode assez novatrice, bien dans la veine de l'Oulipo. ${ }^{19}$ Il a ajouté un appendice aux textes traduits dans lequel il propose des exercices de styles de sa propre invention. Ce qui est absolument justifié, considérant le caractère ouvert du recueil de Queneau. Bognár propose même une parodie d'un style baptisé « de Queneau », qui imite le jargon de Zazie dans le métro.

La traduction d'un texte écrit par un auteur oulipien suppose une réflexion préalable : l'ouvrage en question est-il écrit avec contraintes ? Ces dernières 
sont-elles explicites ou pour le moins facilement identifiables ? On ne peut commencer à chercher des solutions convenables au projet de traduction qu'après avoir répondu à ces questions. ${ }^{20}$ Notons que dans un texte composé par un oulipien, les contraintes peuvent être avouées, cachées, niées ou absentes.

Au sein de l'Oulipo, il existe deux tendances. Certains auteurs préfèrent composer à partir de contraintes explicites, annoncées au préalable (comme Jacques Jouet). D'autres préfèrent dissimuler les contraintes choisies ou du moins que ces dernières ne puissent être décryptées que d'une façon aléatoire (Georges Perec, par exemple). Ainsi est-il souvent difficile de déterminer s'il existe vraiment des contraintes à respecter dans une traduction en projet. Dans un documentaire de Bernard Queysanne, deux traducteurs de La vie mode d'emploi de Georges Perec ont présenté leurs démêlées avec l'ouvrage. ${ }^{21}$ Eugen Helmlé et David Bellos ont témoigné de leurs difficultés à saisir toutes les contraintes cachées dans l'ouvrage, en ajoutant qu'une nouvelle traduction en allemand et en anglais serait nécessaire après la découverte de tout ce qui leur était resté inconnu à cet égard; ce qui se produira certainement, tôt ou tard.

Traduire est une activité difficile, disserter sur une traduction à accomplir l'est tout autant, si l'on ne sait pas quelles sont les contraintes de l'œuvre en question. Le texte oulipien peut être une véritable énigme et son auteur un sphinx. ${ }^{22}$ Le célèbre " principe de Roubaud », respecté par la plupart des textes oulipiens, exige qu'un texte composé à partir d'une contrainte choisisse pour sujet cette contrainte : mais que se passe-t-il en cas de contraintes multiples ? Et si la contrainte correspond justement au fait qu'elle ne doit pas être révélée de façon explicite?

\section{Traduire Jacques Roubaud}

Le cas de Jacques Roubaud est particulièrement complexe. Auteur éminemment oulipien, il travaille très souvent avec des contraintes et souvent sur des formes fixes. Parfois, ses contraintes concernent essentiellement la façon de composer; le résultat sera traduisible sans la nécessité de compromis notable quant à la forme, comme dans le cas du Grand incendie de Londres. Toutefois, ce livre lui-même présente un certain nombre de difficultés. ${ }^{23}$ Au cours de ce long récit, Roubaud a fait appel à dix styles différents, empruntés à Kamo no Chomei, qu'il explique en détail dans le corps du texte. Toutefois, il ne précise nullement le lieu des transitions et l'ordre de succession. Le traducteur hongrois, Csaba Szigeti, affirme avoir identifié les styles correspondants à chaque chapitre et les avoir fidèlement reproduits, mais, comme Roubaud, il ne souhaite pas indiquer les lieux textuels qui leur correspondent.

La tâche est encore plus compliquée pour la traduction d'autres ouvrages Jacques Roubaud refuse de révéler quelque règle de l'écriture qu'il a utilisées, dont la 
connaissance constituerait pourtant une aide précieuse pour le traducteur. Dans ces conditions, les solutions adoptées par un traducteur peuvent être excellentes, mais le doute persiste : a-t-il identifié toutes les contraintes cachées ? N'est-il pas passé à côté d'un principe d'organisation qu'il eût été nécessaire de reproduire dans la traduction?

J'ai dû me confronter à ces difficultés lors de la traduction de Quelque chose noir, recueil de poèmes de Jacques Roubaud. ${ }^{24}$ Il s'agit d'un livre dont l'auteur affirme qu'il est composé selon une contrainte simple : neuf cycles de neuf poèmes de neuf séquences (strophes, vers). Toutefois, un poème a été ajouté à la fin et certains textes contiennent moins de neuf vers ; ainsi cette règle de composition est-elle employée avec quelques clinamens. Jacques Roubaud affirme également qu'il avait souhaité au départ composer un livre selon un plan très strict, avec des contraintes fortes, mais qu'il a renoncé à ce projet en cours de route et qu'il a finalement décomposé la structure ressentie comme trop rigide, indigne du sujet. De cette structure, il ne reste que des traces, des bribes, des éléments épars dans le livre. C'est ainsi cette technique de la décomposition de la forme qui se fait sentir dans beaucoup de poèmes. Méditation à l'identique est, par exemple, un sonnet particulier que la mise en page a réparti en neuf segments, la décomposition autorisant seulement quelques rimes. Énigme, composé à partir d'un sonnet d'Abraham de Vermeil, conserve aussi les traces des rimes (dont quelques-unes sont issues de l'original). Ce poème est composé en alexandrins, procédé exceptionnel dans le recueil. Il faut impérativement maintenir dans la traduction de ces poèmes leur caractère fragmenté, décomposé. De plus, la tâche est parfois plus compliquée. Par exemple, Un jour de juin recompose un poème de Georges Perec ; l'emprunt est souligné dans la dédicace. Il s'agit d'un « beau présent », composé par Perec à l'occasion du mariage de Jacques Roubaud avec Alix-Cléo Blanchette. Le beau présent est un genre inventé par Perec. L'épithalame oulipien, qui est un sous-genre du beau présent, se compose toujours des lettres contenues dans les noms des époux. Jacques Roubaud reprend ainsi plusieurs vers et expressions du poème de Perec, mais il ne construit pas un beau présent parfait : il utilise également quelques lettres interdites par la règle. Confronté à ce texte, le traducteur peut choisir de rendre uniquement le sens de l'original, ou bien il peut essayer de composer un poème observant la même contrainte que Perec, avec quelques clinamens. Et c'est probablement cette deuxième méthode qui est la plus fidèle à l'original, même, éventuellement, au détriment de sa signification primaire. Il serait probablement une très mauvaise solution de composer un beau présent fidèle et parfait, sans transgresser la règle du genre.

Le livre de Roubaud est la cause d'un autre casse-tête pour le traducteur qui s'y aventure, par son usage particulier signes de ponctuation. Les points et les virgules, de même que les blancs longs de la typographie qui apparaissent aussi dans d'autres ouvrages de Roubaud, favorisent la segmentation d'une poésie conçue 
également comme orale. Le traducteur doit respecter ces indices tout en les adaptant à la syntaxe de sa propre langue, ce qui ne va pas sans difficulté. Il est nécessaire de témoigner d'une confiance extrême envers la mise en page originale, avec l'espoir que le défaut d'un point en fin de vers ou la présence d'une virgule à un endroit inattendu témoignent non de la négligence d'un typographe, mais bien de la volonté de l'auteur. D'autre part, une fidélité excessive conduirait au fétichisme, ce qui ne serait pas sans rapport, d'ailleurs, avec l'état d'incertitude relative dans lequel se trouve le traducteur, s'il ne connaît pas toutes les règles de composition employées dans l'original. Ce dernier doit trouver le principe qui a dirigé les choix de l'auteur pour l'adapter ensuite aux possibilités offertes par sa langue et le matériel constitué par le texte source. S'il le peut : c'est pourquoi la traduction ressemble souvent à la tentative de résoudre une énigme.

En fin de compte, par le concept de la potentialité, l'Oulipo réaffirme la pluralité de la littérature. Plusieurs traductions peuvent être bonnes, satisfaisantes et ne trahir en rien l'original en procédant de façons variées. D'ailleurs, une traduction sera d'autant meilleure qu'elle est multiple : tous les ouvrages devraient être traduits, retraduits selon des approches différentes, avec des contraintes différentes, dans le vain espoir d'épuiser les sens potentiels de l'œuvre d'art. Ces solutions, qui ne se veulent pas définitives, reflètent telle ou telle facette de l'original. Une politique éditoriale favorisant cette liberté permettrait d'espérer un plus grand enrichissement des littératures par le biais de la traduction.

\section{Notes}

1 La littérature consacrée à l'Oulipo remplit désormais des bibliothèques entières, avec les ouvrages des Oulipiens eux-mêmes, mais aussi beaucoup d'autres écrits par des spécialistes ; en outre, nous conseillons au lecteur désireux d'acquérir plus d'information de consulter le site internet de l'Oulipo, www.oulipo.net.

2 Quelques articles récents sur différents aspects de la traduction des textes oulipiens : Kate Briggs, "Translation and the Lipogram », Paragraph, 29/3 (2006), 43-54 ; Peter France, « The Rhetoric of Translation », The Modern Language Review, 100 (2005), 255-68; Alison James, «The Maltese and the Mustard Fields: Oulipian Translation », SubStance, 37/1 (2008), $134-47$.

3 La première a été inventée par Michèle Audin, la seconde par Raymond Queneau. Voir le site de l'Oulipo.

4 János Sylvester fut le premier à composer des distiques (hexamètre plus pentamètre) dans l'introduction à sa traduction du Nouveau Testament parue en 1541. Il a rédigé des résumés des Évangiles dans cette forme afin de prouver que la valeur de la langue hongroise n'était pas inférieure à celle des langues anciennes.

5 Antal Radó, A magyar müforditás története 1772-1831, Budapest, Révai Testvérek, 1883, 42-3. Radó y fait surtout l'éloge de sa propre traduction des Odes d'Horace. 
6 Albert Szenci Molnár écrit cela dans sa préface aux Psaumes, édités en 1607, cité par Ildikó Józan : « Irodalom és fordítás », dans A magyar irodalom történetei 3. 1920-tól napjainkig, éd. Mihály Szegedy-Maszák, András Veres, Budapest, Gondolat, 2007, 54. Comme il s'agissait d'un texte sacré, sa volonté de respecter surtout le contenu de l'original était tout à fait naturelle.

7 Justement, le livre de Radó déjà cité.

8 Pour les mètres antiques, voir László Rónay : Az antik metrumok fordítása, dans $A$ müforditás ma, Budapest, Gondolat, 1981, 329-346.

9 Pour les formes encore plus compliquées, comme les cansos des troubadours ou celles de Pétrarque (y compris ses sextines), il a fallu attendre encore quelques décennies. Sándor Weöres est le meilleur exemple de l'adaptation initiée par la traduction, employée par la suite dans sa propre pratique poétique «Bartal ». Quelque peu avant, la célèbre couronne de sonnets d'Attila József avait présenté un mode de découverte de la composition formelle d'origine médiévale ; ce poème est devenu une source d'inspiration importante pour les générations postérieures, jusqu'à la décomposition de la forme par Dezső Tandori dans son Szonettkosz (le titre est à la fois la forme tronquée de Szonettkoszorú [Couronne de sonnets], et un jeu de mot sur le terme « kosz » qui signifie « souillure »). Poème publié dans Fömü, Budapest, Liget, 1999.

10 Ces techniques sont brièvement présentées dans l'article cité de James : « The Maltese and the Mustard Fields... », 140 sqq. La contrainte S+7, une des plus anciennes de la pratique oulipienne, est en soi une technique de traduction, sauf que le « compositeur » se sert de son dictionnaire (parfois non-bilingue) d'une façon particulière.

11 Nous n'abordons pas ici la question délicate des textes non-oulipiens d'auteurs oulipiens, parce que même des plus avertis que nous se demandent si l'on peut vraiment affirmer l'existence de cette catégorie ; voir la note 20 de cet article.

12 La traduction japonaise est due à Shuichiro Shiotsuka.

13 James 135-138. Selon elle, la méthode de Lee « is closer to Perec's linguistic procedures, for example by taking account of supplementary constraints » 138 .

14 Ibid. La référence concerne le livre d'Antoine Berman : La traduction de la lettre ou l'Auberge du lointain, Paris, Seuil, 1999.

15 Hervé Le Tellier, L'esthétique de l'Oulipo, $2^{\mathrm{e}}$ édition, Bordeaux, Le Castor Astral, 2006, 247.

16 Afin de remédier, au moins symboliquement, à cette fâcheuse négligence de La disparition, je propose ici (par métonymie) un titre pour son éventuelle version hongroise : " A hiány ».

17 Ajoutons que l'œuvre narrative de Georges Perec est abondamment traduite en hongrois, malgré l'absence de ses deux grands romans, La Disparition et La Vie mode d'emploi.

18 Voir, à titre d'exemple, le poème de Mónika Mesterházi : Változatok jókívánságra, Szabolcsnak, dans Élet és Irodalom, 54/11, mars 2010.

19 Raymond Queneau, Stílusgyakorlatok, traduits et complétés par Róbert Bognár, Budapest, Helikon, 1988.

20 Jacques Jouet, With (and Without) Constraints, traduit par Roxanne Lapidus, SubStance, Vol. 30, issue $n^{\circ} 96$ (2001), 4-16. Par ailleurs, dans sa conclusion, Jacques Jouet n'est pas véritablement favorable à l'affirmation qu'un texte composé par un Oulipien peut véritablement être sans contrainte. Certains procédés d'écriture qui ne sont pas forcément systématiques, ajoute-t-il, risquent de devenir chez eux des contraintes de création valables pour des ouvrages postérieurs.

21 Lire / traduire Georges Perec : Film documentaire sur la traduction de Georges Perec, écrit et réalisé par Bernard Queysanne, 1999. 
22 Voir encore les variations de Jacques Jouet sur le thème du sphinx et d'CEdipe ainsi que son article précité.

23 Jacques Roubaud, Le Grand Incendie de Londres, Paris, Seuil, 1989. Il s'agit du premier volume d'une œuvre en six "branches" que Jacques Roubaud appelle son "Projet".

24 Jacques Roubaud, Quelque chose noir, Paris, Gallimard, 1986. En hongrois : Jacques Roubaud, Vala mi: fekete, traduit par Levente Seláf, Bratislava, Kalligram, 2010. 